\title{
Introduction to the special issue on cyber security and management
}

\author{
Frederick T. Sheldon • J. Todd McDonald
}

Published online: 26 October 2012

(C) Springer-Verlag Berlin Heidelberg (outside the USA) 2012

Today's cyberspace is a powerful, virtual environment enabled by a pervasive global digital infrastructure. Even though it offers an ideal landscape for the conduct of commerce, science, education, communication, and government, cyberspace remains vulnerable to attack and manipulation from ever-evolving malicious threats. Cyber security, like other research disciplines, must often draw upon disparate fields of study to advance and create applied security measures that ensure adequate levels of confidentiality, integrity, and availability.

Researchers often face challenging problems in the engineering and science fields that are only uniquely addressed by applying theoretical and practical results from different scientific areas of study. The pervasiveness of the cyber domain in almost every aspect of modern life, from government to private sector, has brought the issue of security research squarely to the forefront of international attention. As such, the need for multidisciplinary approaches to help solve the hard problems of security has become paramount as well.

The impact of malicious cyber attacks on various commercial enterprises and military systems can now reach catastrophic proportions in terms of financial loss and compromise of critical information. Lockheed Martin, RSA, Google, Citigroup, the International Monetary Fund, MasterCard, the Unites States (US) Chamber of Commerce, and the US Department of Defense have all reported breech and infiltration through hackers in the last two years (Johnson 2011; Shachtman 2011; Wyler 2011; Thomas and Katrandjian 2011; Hack Against Citigroup revealed: is bank security system really bullet-proof? International Business Times New York, Jun. 09 2011). In 2011, 70 million Sony PlayStation users had their credit card

\section{F. T. Sheldon ( $\square)$}

Oak Ridge National Laboratory, Oak Ridge, TN, USA

e-mail: sheldonft@ornl.gov 
information compromised (Johnson 2011). In 2009, the worldwide ATM Industry Association reported over $\$ 1$ billion in annual global losses from credit card fraud and electronic crime associated with ATMs (Siciliano 2009). In 2011, the Federal Bureau of Investigation's Internet Crime Complaint Center (IC3) reported that it had received 314,246 complaints with a reported dollar loss of \$485.3 million (Internet Crime Report). Richard Clarke, the former US government security chief that prognosticated the 9/11 attacks on the US, asserted recently (Waugh 2012) that every major company in the United States has already been penetrated by Chinese hackers looking to steal military and financial secrets. Finally, according to the 2012 Norton Cybercrime Report, in the United States, cybercrime costs consumers \$21 billion a year. Globally, cybercrime cost consumers $\$ 110$ billion over the past 12 months ( $\$ 388$ billion including lost productivity), with over 1.5 million cybercrime victims a day (Norton 2012).

The state of the art for research and realized solutions is often outpaced by the sophistication and number of attacks carried out by nation-state and organized cyber-criminals. In cyber security, we can look historically to see how the marriage of multiple disciplines has allowed novel insight in the advancement of both theoretical results and practical applications. In 1882, a telegraph engineer named Frank Miller first described the concept of a one-time pad (OTP) data cipher (Miller 1882), where ciphertext is created by modular addition of plaintext and key material of equal length (Vernam 1926). In 1918, AT\&T Bell Labs engineer Gilbert Vernam used the same concept to patent an automated telegraphic switching system where the XOR combining function was implemented through relay switches. In 1948, computer scientist Claude Shannon pioneered the field of information theory based on his wartime research of cryptographic algorithms (Shannon 1948). He proved that the one-time pad implementation was information-theoretically secure by virtue that the entropy between the plaintext and ciphertext was maximal based on mutual information probabilities (Shannon 1949). In essence, OTP is unbreakable even given unlimited computational power. Unfortunately, the one-time pad has the limitation that the key size must be equal to the plaintext size.

Given the fact that the one-time pad is a symmetric key algorithm, transmission of the key between parties is extremely problematic and not practical for common applications. It was not until the last decade that this limitation could be overcome based on breakthroughs in the area of quantum physics. Quantum Key Distribution (Mink et al. 2006), for example, has been realized in physical systems and allows for the transmission of very large keys. Unconditional security of quantum key generation protocols derives from quantum laws, Heisenberg's uncertainty principle, and the fact that quantum information cannot be copied or measured without detection. Quantum cryptography is in the advanced experimental and development stage, and well on its way to full commercial realization in the next decade (McDonald and HivelyLM 2012). Ultimately, a major problem of cyber security (confidentiality in this example) has been advanced through a series of combined work and novel contributions in multiple disparate disciplines. We believe other cyber security hard problems can and will be furthered in a similar manner.

The nature of exceptional research can be characterized by asking exceptional questions that are relevant to current hard problems. In some cases, the solutions are 
best addressed through exceptional management practices, while others are mutidisciplenary and still others are achieved through experimental (empirical validation) means. In this special issue, we provide the following articles that exemplify these cases toward solving the hard problems of Cyber Security and Management:

- Defining and computing a value based cyber-security measure

- Measuring and ranking attacks based on vulnerability analysis

- Behavioral analysis of botnets for threat intelligence

- Management and security of remote sensor networks in hazardous environments using over the air programming

\section{References}

Hack Against Citigroup revealed: is bank security system really bullet-proof? International Business Times New York, Jun 09, 2011. http://newyork.ibtimes.com/articles/160322/20110609/citibankhacked-security-online.htm

Internet Crime Report (2011) U.S. Federal Bureau of Investigation, Internet Crime Complaint Center. http://www.ic3.gov/media/annualreport/2011_IC3Report.pdf

Johnson R (2011) The biggest hacking attacks of 2011. Business Insider, Jun 13, 2011. http://www.businessinsider.com/imf-cyber-attacked-hackers-sony-rsa-lockheed-martin-epsilonmichaels-2011-6?op=1

McDonald JT, HivelyLM (2012) Multidisciplinary approaches for cyber security, Jr. of Computer Engineering and Information Technology http://scitechnol.com/jceithome.php

Miller F (1882) Telegraphic code to insure privacy and secrecy in the transmission of telegrams

Mink A, Tang X, Ma L, Nakassis T, et al (2006) High speed quantum key distribution system supports one-time pad encryption of real-time video. In Donkor EJ, Pirich AR, Brandt HE (eds) Proceeding of SPIE quantum information and computation IV, 6244: $62440 \mathrm{M}$

Norton (2012) Cybercrime Report (Sept. 5, 2012). http://now-static.norton.com/now/en/pu/images/ Promotions/2012/cybercrimeReport/2012_Norton_Cybercrime_Report_Master_FINAL_050912.pdf

Shachtman N (2011) Computer virus hits US drone fleet. Wired, Oct 7, 2011. http://www.wired.com/ dangerroom/2011/10/virus-hits-drone-fleet/

Shannon C (1948) A mathematical theory of communications. Bell Syst Tech J 27: 379-423 \& 623-656

Shannon C (1949) Communication theory of secrecy systems. Bell Syst Tech J 28(4):656-715

Siciliano R (2009) Credit card fraud tops consumers concerns. Finextra, Apr 7, 2009. http://www. finextra.com/community/fullblog.aspx?id=2756

Thomas P, Katrandjian O (2011) Chinese hack into us chamber of commerce, authorities say. ABC News, Dec 21, 2011. http://abcnews.go.com/International/chinese-hack-us-chamber-commerce-authorities/ story?id=15207642

Vernam G (1926) Cipher printing telegraph systems for secret wire and radio telegraphic communications. J IEEE 55:109-115

Waugh R (2012) Cyber-espionage warning from US security chief who warned of 9/11. Daily Mail Online. Mar 28, 2012. http://www.dailymail.co.uk/sciencetech/article-2121624/Every-major-companyU-S-hacked-China-Cyber-espionage-warning-U-S-security-chief-warned-9-11.html

Wyler G (2011) Pentagon admits 24,000 files were hacked, declares cyberspace a theater of war. Business Insider, Jun. 13, 2011. http://articles.businessinsider.com/2011-07-14/politics/30034321_1_cyberthreat-cyber-attacks-new-command 